\title{
Vertex and Edge Hyper Wiener Number of Some Cluster Graphs
}

\author{
K.Thilakam ${ }^{1}$, R.Bhuvaneswari ${ }^{2}$ \\ (PG and Research Department of Mathematics, SeethalakshmiRamaswami College, Thiruchirappali -2, India)
}

\begin{abstract}
One of the most widely known topological descriptors is the Wiener index or (Wiener number) named after American chemist Harold Wiener in 1947. Wiener number of a connected graph $G$ is defined as the sum of the distances between distinct pairs of vertices of G..It correlates between physico- chemical and structural properties. The hyper wiener index denoted by $W W$ of a graph $G$ was introduced by Randic and his definition is applicable to trees only. Klein, Lukovits and Gutman introduced the formula for both trees and cycle containing structures. The Hyper Wiener index is defined as $W W(G)=\left(\sum d^{2}(u, v)+\sum d(u, v)\right) / 2, \quad$ where $d(u, v)$ denotes the distance between the vertices $u$ and $v$ in the graph $G$ and the summations run over all distinct pairs of vertices of G. Recently an edge version of Hyper Wiener Index was introduced by Ali Iranmanesh.In this paper, we have determined Hyper Wiener numbers of some Cluster graphs and also for some bipartite cluster graphs
\end{abstract}

Keywords: distance sum, nanostar, Vertex and edge Wiener index, Vertex and edge Hyper Wiener index

\section{Introduction}

A Graph $G$ is formally defined to be a pair $[\mathrm{V}(\mathrm{G}, \mathrm{E}(\mathrm{G})]$ where $\mathrm{V}(\mathrm{G})$ is a non empty finite set of elements called vertices and $E(G)$ is a finite set of unordered pairs of elements of $V(G)$ called edges.Molecular graphs represent the constitution of molecules[1]. They are generated using the following rule: Vertices stand for atoms and edges for bonds. A graph theoretical distance $\mathrm{d}(\mathrm{u}, \mathrm{v})$ between the vertices $\mathrm{u}$ and $\mathrm{v}$ of the graph $\mathrm{G}$ is equal to the length of the shortest path that connects $u$ and $v$. An invariant of a graph $\mathrm{G}$ is a number associated with $\mathrm{G}$ that has the same value for any graph isomorphic to $\mathrm{G}$. If $\mathrm{G}$ is a molecular graph then the corresponding invariants are called molecular descriptor or topological indices and they are used in theoretical chemistry for the design of so called Quantitative Structure Property Relations (QSPR) and Quantitative Structure Activity Relations(QSAR). One of the oldest topological index is Wiener index and is defined as the half of the sum of all the distances between every pair of vertices of G.[2] ie

$$
\mathrm{W}(\mathrm{G})=\frac{1}{2} \sum_{u, v} d(u, v)
$$

The hyper wiener index was introduced by Randic [3] and his definition is applicable to trees only. Klein, Lukovits and Gutman[4] introduced the formula for both trees and cycle containing structures and Hyper Wiener index WW of a graph $G$ is defined as

$$
W W(G)=\frac{1}{2}\left(\sum d(u, v)+\sum d(u, v)^{2}\right)=\frac{1}{2}\left(W(G)+\sum d(u, v)^{2}\right)
$$

and summation runs over all distinct pairs of vertices. Equations (1) and (2) are called vertex version of Wiener and Hyper Wiener index respectively.The edge versions of Wiener Index were introduced by A.Iranmanesh, I.Gutman, O.Khormali,A.Mahmiani in 2009 [5] and is defined as follows The first edge- Wiener number is

$$
\begin{aligned}
& W_{e 0}(G)=\frac{1}{2} \sum_{\substack{e, f \in E(G) \\
e \neq f}} d_{0}(e, f) \\
& \text { Where } \mathrm{d}_{0}(\mathrm{e}, \mathrm{f})=\mathrm{d}_{1}(\mathrm{e}, \mathrm{f})+1 \quad \mathrm{e} \neq \mathrm{f} \\
& \mathrm{d}_{1}(\mathrm{e}, \mathrm{f})=\min \{\mathrm{d}(\mathrm{x}, \mathrm{u}), \mathrm{d}(\mathrm{x}, \mathrm{v}), \mathrm{d}(\mathrm{y}, \mathrm{u}), \mathrm{d}(\mathrm{y}, \mathrm{v})\} \text { such that } \mathrm{e}=\mathrm{xy} \text { and } \mathrm{f}=\mathrm{uv} \text { and } \mathrm{W}_{\mathrm{e} 0}=\mathrm{W}(\mathrm{L}(\mathrm{G})) \text {. }
\end{aligned}
$$

The second edge - Wiener number is

$$
\begin{aligned}
& W_{e 4}(G)=\frac{1}{2} \sum_{\substack{e, f \in E(G) \\
e \neq f}} d_{4}(e, f) \\
& \text { Where } \mathrm{d}_{4}(\mathrm{e}, \mathrm{f})=\left\{\begin{array}{ccc}
\mathrm{d}_{2}(\mathrm{e}, \mathrm{f}) & \mathrm{e} \neq \mathrm{f} \\
& = & \mathrm{e}=\mathrm{f}
\end{array}\right.
\end{aligned}
$$

$d_{2}(e, f)=\max \{d(x, u), d(x, v), d(y, u), d(y, v)\}$ such that $e=x y$ and $f=u v$. The edge version of Hyper Wiener indices are defined as[6]

$$
W W_{e i}(G)=W_{e i}(G)+W_{e i}^{d^{2}}(G)(5)
$$




$$
W_{e i}^{d^{2}}(G)=\frac{1}{2} \sum_{\substack{e, f \in E(G) \\ e \neq f}} d_{i}^{2}(e, f), i=0,4
$$

where $d_{i}$ are distances for $i=0,4$. Since all these topological indices are depends on distance between every pair of vertices of a given graph G.The graphs with large number of edges are referred as graph representations of inorganic clusters, so-called cluster graphs. In this paper we have determined vertex and edge hyper Wiener number of some cluster graphs.

\section{Some Known Results:}

For $\mathrm{K}_{\mathrm{n}}$

$$
\begin{aligned}
& \text { 1. } W\left(K_{n}\right)=\frac{n(n-1)}{2} \\
& \text { 2. } W\left(K_{n}\right)=\frac{n(n-1)}{2} \\
& \text { 3. } W_{e 0}\left(K_{n}\right)=\frac{n(n-1)^{2}(n-2)}{4} \\
& \text { 4. } W W_{e 0}\left(K_{n}\right)=\frac{n((n-1)(n-2)(3 n-5)}{4} \\
& \text { 5. } W_{e 4}\left(K_{n}\right)=\frac{n(n-1)(n-2)(n+1)}{8} \\
& \text { For } \mathrm{K}_{\mathrm{n}, \mathrm{m}} \\
& \text { 6. } W_{e 4}\left(K_{n}\right)=\frac{n(n-1)(n-2)(n+1)}{4}
\end{aligned}
$$

1. $\mathrm{W}\left(\mathrm{K}_{\mathrm{n}, \mathrm{m}}\right)=(\mathrm{m}+\mathrm{n})^{2}-(\mathrm{m}+\mathrm{n})-\mathrm{mn}$

2. $\mathrm{WW}\left(\mathrm{K}_{\mathrm{n}, \mathrm{m}}\right)=3(\mathrm{~m}+\mathrm{n})^{2}-3(\mathrm{~m}+\mathrm{n})-4 \mathrm{mn}$

3. $\mathrm{W}_{\mathrm{e} 0}\left(\mathrm{~K}_{\mathrm{n}, \mathrm{m}}\right)=\frac{n m}{2}(2 \mathrm{~nm}-\mathrm{n}-\mathrm{m})$

4. $\mathrm{WW}_{\mathrm{e} 0}\left(\mathrm{~K}_{\mathrm{n}, \mathrm{m}}\right)=\mathrm{nm}(3 \mathrm{~nm}-2 \mathrm{n}-2 \mathrm{~m}+1)$

5. $\mathrm{W}_{\mathrm{e} 4}\left(\mathrm{~K}_{\mathrm{n}, \mathrm{m}}\right)=\mathrm{nm}(\mathrm{nm}-1)$

6. $\mathrm{WW}_{\mathrm{e} 4}\left(\mathrm{~K}_{\mathrm{n}, \mathrm{m}}\right)=3 \mathrm{~nm}(\mathrm{~nm}-1)$

\section{Cluster Graphs Obtain From Complete Graphs}

I.Gutman and L.Pavlovic [7] introduced four classes of graphs obtained from complete graph by deleting edges. When the edges are deleted from $K_{n}$, in a random manner, one-by-one, then the vertex version of Wiener numbers increases and edge version of Wiener indices will decreased .

DEFINITION 2.1: Let $\mathrm{v}$ be a vertex of the complete graph $\mathrm{K}_{\mathrm{n}}, \mathrm{n} \geq 3$ and let $\mathrm{e}_{\mathrm{i}}, \mathrm{i}=1,2, \ldots \mathrm{k}, 1 \leq \mathrm{k} \leq \mathrm{n}-1$, be its distinct edges, all being incident to $\mathrm{v}$. The graph $\mathrm{Ka}_{\mathrm{n}}(\mathrm{k})$ is obtained by deleting $\mathrm{e}_{\mathrm{i}}, \mathrm{i}=1,2, . ., \mathrm{k}$, from $\mathrm{K}_{\mathrm{n}}$. In addition, $\mathrm{Ka}_{\mathrm{n}}(0) \equiv \mathrm{K}_{\mathrm{n}}$.

DEFINITION 2.2:Let $\mathrm{f}_{\mathrm{i}}, \mathrm{i}=1,2, \ldots, \mathrm{k}, 1 \leq \mathrm{k} \leq\left\lfloor\frac{n}{2}\right\rfloor$ be independent edges of the complete graph $K_{n}, n \geq 3$. The graph $K b_{n}(k)$ is obtained by deleting $f_{i}, i=1,2, \ldots$, from $K_{n}$. In additionK $b_{n}(0) \equiv$ $\mathrm{K}_{\mathrm{n}}$.

DEFINITION 2.3: Let $\mathrm{V}_{\mathrm{k}}$ be a k-element subset of the vertex set of thecomplete graph $\mathrm{K}_{\mathrm{n}}, 2 \leq \mathrm{k} \leq \mathrm{n}, \mathrm{n} \geq 3$. The graph $\mathrm{Kc}_{\mathrm{n}}(\mathrm{k})$ is obtained by deleting from $\mathrm{K}_{\mathrm{n}}$ all the edges connecting pairs of vertices from $\mathrm{V}_{\mathrm{k}}$. In addition, $\operatorname{Kc}_{\mathrm{n}}(0)=\mathrm{Kc}_{\mathrm{n}}(1)=\mathrm{K}_{\mathrm{n}}$.

\section{Theorem 2.1}

$\mathrm{W}\left(\mathrm{Ka}_{\mathrm{n}}(\mathrm{k})\right)=\left(\begin{array}{l}n \\ 2\end{array}\right)+\mathrm{k}, 1 \leq \mathrm{k}<\mathrm{n}-1$

\section{Theorem:2.2}

$$
\begin{array}{ll}
\text { I. } & \mathrm{WW}\left(\mathrm{Ka}_{\mathrm{n}}(\mathrm{k})\right)=\mathrm{n}(\mathrm{n}-1)+4 \mathrm{k} \\
\text { II. } & \mathrm{W}_{\mathrm{eo}}\left(\mathrm{Ka}_{\mathrm{n}}(\mathrm{k})\right)=\frac{(n-1)(n-2)}{4}(n(n-1)-4 k)+\left(\begin{array}{l}
k \\
2
\end{array}\right) \\
\text { III. } & \mathrm{WW}_{\mathrm{eo}}\left(\mathrm{Ka}_{\mathrm{n}}(\mathrm{k})\right)=\frac{n(n-1)(n-2)(3 n-5)}{4}-k\left(3 n^{2}-11 n+10\right)+\mathrm{k}(\mathrm{k}-1) \\
\text { IV. } & \mathrm{W}_{\mathrm{e} 4}\left(\mathrm{Ka}_{\mathrm{n}}(\mathrm{k})\right)=\frac{n(n-1)(n-2)(n+1)}{8}-k\left(\frac{n(n-1)}{2}-1\right)+\left(\begin{array}{l}
k \\
2
\end{array}\right)
\end{array}
$$


V. $\quad \mathrm{WW}_{\mathrm{e} 4}\left(\mathrm{Ka}_{\mathrm{n}}(\mathrm{k})\right)=\frac{n(n-1)(n-2)(n+1)}{4}-2 k\left(\frac{n(n-1)}{2}-1\right)+k(k-1)$

Proof:

I. Let $\mathrm{v}$ be any vertex of $\mathrm{K}_{\mathrm{n}}$. In $\mathrm{K}_{\mathrm{n}}$ each vertex has $\mathrm{n}-1$ edges incident with it . If we remove $\mathrm{k}$ edges from $\mathrm{v}$ where $1 \leq \mathrm{k}<\mathrm{n}-1, \mathrm{k}$ pairs of vertices are of distance 2 . Total number of pairs of vertices of $\mathrm{K}_{\mathrm{n}}$ is $\left(\begin{array}{l}n \\ 2\end{array}\right)$

Hence $\left(\begin{array}{l}n \\ 2\end{array}\right)$-k pairs of vertices are of distance 1.Therefore

$$
\sum_{\substack{i, j=1 \\
i<j}}^{n} d^{2}\left(v_{i}, v_{j}\right)=\left(\left(\begin{array}{l}
n \\
2
\end{array}\right)-k\right) \cdot 1+k(2)^{2}=\left(\begin{array}{l}
n \\
2
\end{array}\right)-k+4 k
$$

Hence $\mathrm{WW}\left(\mathrm{Ka}_{\mathrm{n}}(\mathrm{k})\right)=\mathrm{W}\left(\mathrm{Ka}_{\mathrm{n}}(\mathrm{k})\right)+\sum_{\substack{i, j=1 \\ i<j}}^{n} d^{2}\left(v_{i}, v_{j}\right)=\left(\begin{array}{l}n \\ 2\end{array}\right)+\mathrm{k}+\left(\begin{array}{l}n \\ 2\end{array}\right)-k+4 k=\mathrm{n}(\mathrm{n}-1)+4 \mathrm{k}$.

II. Let $\mathrm{E}\left(\mathrm{K}_{\mathrm{n}}\right)=\left\{\mathrm{e}_{21}, \mathrm{e}_{31}, . . \mathrm{e}_{\mathrm{i} 1} . . \mathrm{e}_{\mathrm{n} 1} ; \mathrm{e}_{32}, \mathrm{e}_{42}, . . \mathrm{e}_{\mathrm{i} 2} . . \mathrm{e}_{\mathrm{n} 2} ; \mathrm{e}_{43}, \mathrm{e}_{53} . . \mathrm{e}_{\mathrm{i} 3} . . \mathrm{e}_{\mathrm{n} 3} ; \ldots . \mathrm{e}_{\mathrm{i}+1 \mathrm{i}}, \mathrm{e}_{\mathrm{i}+2 \mathrm{i}} \ldots \mathrm{e}_{\mathrm{ni}} ; \mathrm{e}_{\mathrm{nn}-\mathrm{i}}\right\}$.

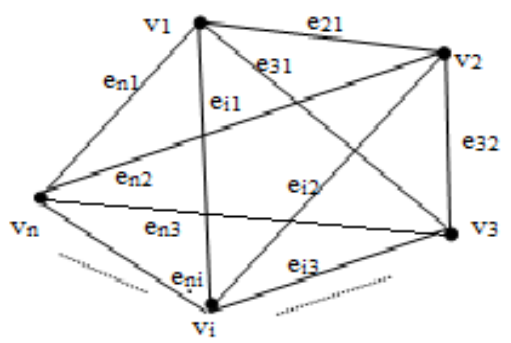

Figure 1.1

First edge - distance matrix $D_{1}=\left(d_{i j}\right)_{\frac{n(n-1)}{2}} \times \frac{n(n-1)}{2}$ of Kn is given below.

$\begin{array}{llllllllllllllll}e_{21} & e_{31} \ldots e_{i 1} \ldots & e_{n 1} & e_{32} & e_{42} \ldots & e_{i 2} \ldots & e_{n 2}^{2} & e_{43}{ }^{2} & e_{53} \ldots & e_{i 3} \ldots & e_{n 3} & \ldots & e_{i+1 i} & e_{i+2 i} \ldots e_{n i} \ldots & e_{n n-1}\end{array}$

$$
\begin{array}{llllllllllllllll}
e_{21} 0 & 1 & 1 & 1 & 1 & 1 & 1 & 1 & 2 & 2 & 2 & 2 & 2 & 2 & 2 & 2 \\
e_{31} 1 & 0 & 1 & 1 & 1 & 2 & 2 & 2 & 1 & 1 & 1 & 1 & 2 & 2 & 2 & 2
\end{array}
$$

$\cdot$

$\begin{array}{llllllllllllllll}e_{i 1} 1 & 1 & 0 & 1 & 2 & 2 & 1 & 2 & 2 & 2 & 1 & 2 & 1 & 1 & 1 & 2\end{array}$

$\begin{array}{ccccccccccccccccc} & e_{n 1} 1 & 1 & 1 & 0 & 2 & 2 & 2 & 1 & 2 & 2 & 2 & 1 & 2 & 2 & 1 & 1 \\ e_{32} 1 & 1 & 2 & 2 & 0 & 1 & 1 & 1 & 1 & 1 & 1 & 1 & 2 & 2 & 2 & 2 & \\ e_{42} 1 & 2 & 2 & 2 & 1 & 0 & 1 & 1 & 1 & 2 & 2 & 2 & 2 & 2 & 2 & 2\end{array}$

$$
\begin{array}{lllllllllllllllll}
e_{i 2} 1 & 2 & 2 & 2 & 1 & 1 & 0 & 1 & 2 & 2 & 1 & 2 & 1 & 1 & 1 & 2
\end{array}
$$

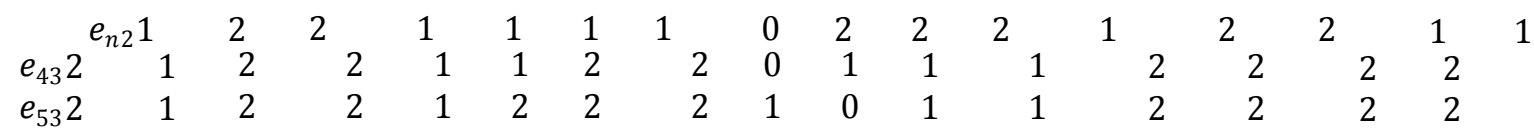

$$
\begin{array}{lllllllllllllllllll}
e_{i 3} 2 & 1 & 1 & 2 & 1 & 2 & 1 & 2 & 1 & 1 & 0 & 1 & 1 & 1 & 1 & 2 \\
\cdot & & & & & & & & & & & & & & & & & & \\
e_{n 3} 2 & 1 & 2 & & 1 & 1 & 2 & 2 & & 1 & 1 & 1 & 1 & 0 & 2 & 2 & 1 & 1
\end{array}
$$




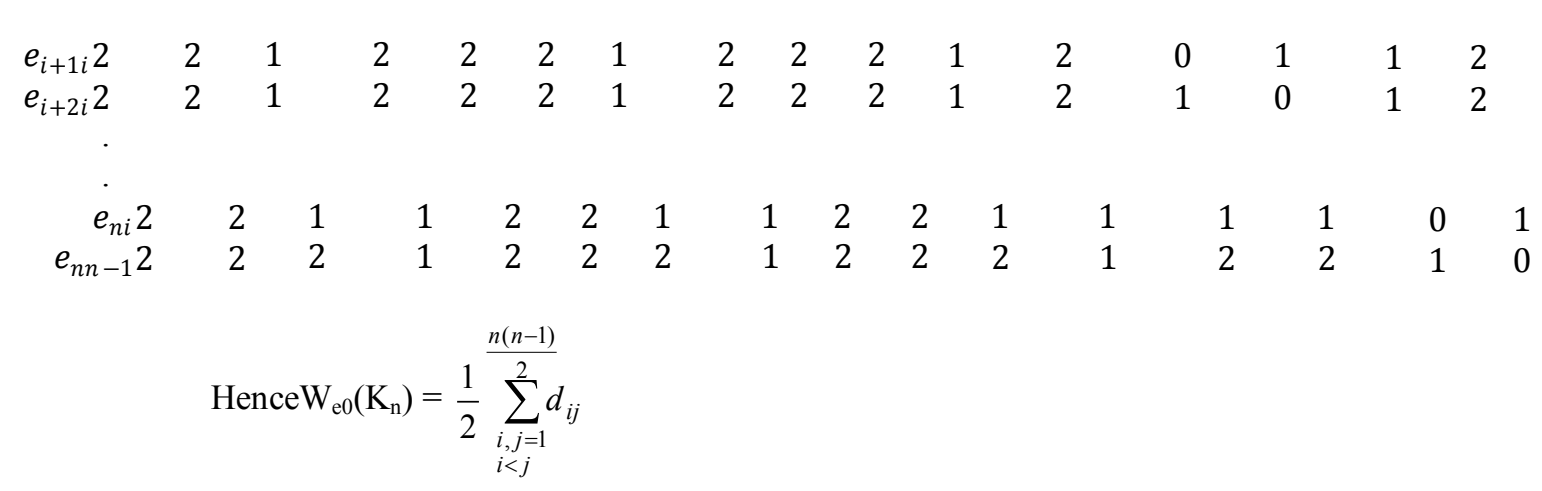

In $K_{n}$ each vertex has $n-1$ edges incident to it. Removal of an edge from $K_{n}$ will remove the corresponding row and column from $\mathrm{D}_{1}$. But removal of more than one edge will remove the common entries twice. Let $\mathrm{G}$ be a graph obtain from $\mathrm{K}_{\mathrm{n}}$ by deleting some edges from $\mathrm{K}_{\mathrm{n}}$. Then

$$
\begin{aligned}
& \left.W e_{i}(G)=W e_{i}\left(K_{n}\right)-(\text { Number of removed edges })(\text { Row (column }) \text { sum }\right)+ \text { Sum } \\
& \text { of common entries }, i=0,4
\end{aligned}
$$

For $\mathrm{Ka}_{\mathrm{n}}(\mathrm{k})$,number of edges removed is $\mathrm{k}$ and row(column) sum of $\mathrm{D}_{1}$ is $\mathrm{n}(\mathrm{n}-1)-2 \mathrm{n}+2$, since number of 2's in each row(column) is $\frac{n(n-1)}{2}-1-2(n-2)$ and number of 1 's is $\frac{n(n-1)}{2}-1-\left(\frac{n(n-1)}{2}-1-2(n-2)\right)$ and the sum of common entries are $\left(\begin{array}{l}k \\ 2\end{array}\right)$ Therefore from (i )

$$
\begin{aligned}
\left.\mathrm{W}_{\mathrm{eo}}\left(\mathrm{Ka}_{\mathrm{n}}(\mathrm{k})\right)=W_{e 0}\left(K_{n}\right)-k(n(n-1)-2 n+2)\right\}+\left(\begin{array}{l}
k \\
2
\end{array}\right) \\
\text { Hence } \quad \mathrm{W}_{\mathrm{eo}}\left(\mathrm{Ka}_{\mathrm{n}}(\mathrm{k})\right)=\frac{(n-1)(n-2)}{4}(n(n-1)-4 k)+\left(\begin{array}{l}
k \\
2
\end{array}\right)
\end{aligned}
$$

III. Let $\mathrm{G}$ be a graph obtain from $\mathrm{K}_{\mathrm{n}}$ by deleting some edges from $\mathrm{K}_{\mathrm{n}}$. Then

$$
\begin{gathered}
W_{e i}^{d^{2}}(G)=W_{e i}^{d^{2}}\left(K_{n}\right)-(\text { Number of removed edges)(Sum of square of entries } \\
\text { in a Row }(\text { column }))+ \text { Sum of square of common entries }, i=0,4
\end{gathered}
$$

Since

$$
W_{e i}^{d^{2}}\left(K_{n}\right)=W W_{e i}\left(K_{n}\right)-W_{e i}\left(K_{n}\right), i=0,4
$$

For $\mathrm{Ka}_{\mathrm{n}}(\mathrm{k})$,sum of square of entries in a row (column) of $\mathrm{D}_{1}$ is $2 \mathrm{n}(\mathrm{n}-1)-6(\mathrm{n}-2)-4$ and sum of square of common entries is $\left(\begin{array}{l}k \\ 2\end{array}\right)$.Therefore from (ii) we get,

$$
W_{e 0}^{d^{2}}\left(K a_{n}(\mathrm{k})\right)=W_{e 0}^{d^{2}}\left(K_{n}\right)-k\{2 \mathrm{n}(\mathrm{n}-1)-6(\mathrm{n}-2)-4\}+\left(\begin{array}{c}
k \\
2
\end{array}\right)
$$

Therefore $\mathrm{WW}_{\text {eo }}\left(\mathrm{Ka}_{\mathrm{n}}(\mathrm{k})\right)=\frac{n(n-1)(n-2)(3 n-5)}{4}-k\left(3 n^{2}-11 n+10\right)+\mathrm{k}(\mathrm{k}-1)$.

IV. Second edge distance matrix $\mathrm{D}_{4}=\left(\right.$ dij) of $\mathrm{K}_{\mathrm{n}}$ is a $\frac{n(n-1)}{2} X \frac{n(n-1)}{2}$ matrix in which all entries are one except the diagonal .Hence row(column) sum of $\mathrm{D}_{4}$ is $\frac{n(n-1)}{2}-1$ and for $\mathrm{Ka}_{n}(\mathrm{k})$ sum of common entries is $\left(\begin{array}{l}k \\ 2\end{array}\right)$.Therefore from (i) we get

$$
\mathrm{W}_{\mathrm{e} 4}\left(\mathrm{Ka}_{\mathrm{n}}(\mathrm{k})\right)=\frac{n(n-1)(n-2)(n+1)}{8}-k\left(\frac{n(n-1)}{2}-1\right)+\left(\begin{array}{l}
k \\
2
\end{array}\right)
$$

V. Sum of square of entries in a row(column) of $\mathrm{D}_{4}$ is $(\mathrm{n}(\mathrm{n}-1) / 2)-1$ and for $\mathrm{Ka}_{\mathrm{n}}(\mathrm{k})$ sum of square of common entries is $\left(\begin{array}{l}k \\ 2\end{array}\right)$.Therefore from (iii) we get

$$
W_{e 0}^{d^{2}}\left(K a_{n}(\mathrm{k})\right)=W_{e 0}^{d^{2}}\left(K_{n}\right)-k\left(\frac{n(n-1)}{2}-1\right)+\left(\begin{array}{l}
k \\
2
\end{array}\right)
$$

Hence the result.

Theorem :2.3 


$$
\mathrm{W}\left(\mathrm{Kb}_{\mathrm{n}}(\mathrm{k})\right)=\left(\begin{array}{l}
n \\
2
\end{array}\right)+\mathrm{k} \quad[\mathbf{8}]
$$

Theorem 2.4
I. $\quad \mathrm{WW}\left(\mathrm{Kb}_{\mathrm{n}}(\mathrm{k})\right)=\mathrm{n}(\mathrm{n}-1)+4 \mathrm{k}$
II. $\quad \mathrm{W}_{\mathrm{eo}}\left(\mathrm{Kb}_{\mathrm{n}}(\mathrm{k})\right)=\frac{(n-1)(n-2)}{4}(n(n-1)-4 k)+k(k-1)$
III. $\quad \mathrm{WW}_{\mathrm{eo}}\left(\mathrm{Kb}_{\mathrm{n}}(\mathrm{k})\right)=\frac{n(n-1)(n-2)(3 n-5)}{4}-k\left(3 n^{2}-11 n+10\right)+3 \mathrm{k}(\mathrm{k}-1)$
IV. $\quad \mathrm{W}_{\mathrm{e} 4}\left(\mathrm{~Kb}_{\mathrm{n}}(\mathrm{k})\right)=\frac{n(n-1)(n-2)(n+1)}{8}-k\left(\frac{n(n-1)}{2}-1\right)+\left(\begin{array}{l}k \\ 2\end{array}\right)$
V. $\quad \mathrm{WW}_{\mathrm{e} 4}\left(\mathrm{~Kb}_{\mathrm{n}}(\mathrm{k})\right)=\frac{n(n-1)(n-2)(n+1)}{4}-2 k\left(\frac{n(n-1)}{2}-1\right)+k(k-1)$

Proof:

As $\mathrm{k}$ independent edges are removed from $\mathrm{K}_{\mathrm{n}}$, there are $\mathrm{k}$ pairs of vertices andthey are of distance 2 and for $K b_{\mathrm{n}}(\mathrm{k})$ sum of common entries is $2\left(\begin{array}{l}k \\ 2\end{array}\right)=\mathrm{k}(\mathrm{k}-1)$ and sum of square of common entries is $4\left(\begin{array}{l}k \\ 2\end{array}\right)=$ $2 k(k-1)$.Hence, the result follows from Theorem 1.2.

Theorem 2.5

$\mathrm{W}\left(\mathrm{Kc}_{\mathrm{n}}(\mathrm{k})\right)=\left(\begin{array}{c}n \\ 2\end{array}\right)+\left(\begin{array}{c}k \\ 2\end{array}\right) 1<\mathrm{k}<\mathrm{n}-1[8]$

Theorem 2.6

I. $\quad \mathrm{W}\left(\mathrm{Kc}_{\mathrm{n}}(\mathrm{k})\right)=\mathrm{n}(\mathrm{n}-1)+2 \mathrm{k}(\mathrm{k}-1)$

II. $\quad \mathrm{W}_{\mathrm{e} 0}\left(\mathrm{Kc}_{\mathrm{n}}(\mathrm{k})\right)=\frac{n(n-1)^{2}(n-2)}{4}-\frac{k(k-1)}{4}\{2(n-1)(n-2)-(k-1)(k-2)\}$

III. $\quad \mathrm{WW}_{\mathrm{e} 0}\left(\mathrm{Kc}_{\mathrm{n}}(\mathrm{k})\right)=\frac{n(n-1)(n-2)(2 n-3)}{4}-\frac{k(k-1)}{4}\left\{6 n^{2}-22 n+10-3 k^{2}+11 k\right\}$

IV. $\quad \mathrm{W}_{\mathrm{e} 4}\left(\mathrm{Kc}_{\mathrm{n}}(\mathrm{k})\right)=\frac{n(n-1)(n-2)(n+1)}{8}-\frac{k(k-1)}{8}\left\{2 n(n-1)-k^{2}+k-2\right\}$

V. $\quad \mathrm{WW}_{\mathrm{e} 4}\left(\mathrm{Kc}_{\mathrm{n}}(\mathrm{k})\right)=\frac{n(n-1)(n-2)(n+1)}{4}-\frac{k(k-1)}{4}\{2 n(n-1)-k(k-1)-2\}$

Proof:

I. If $\mathrm{V}_{\mathrm{k}}=\left\{\mathrm{v}_{1}, \mathrm{~V}_{2}, \ldots, \mathrm{V}_{\mathrm{k}}\right\}$, then the number of edges which are mutually incident

among $\mathrm{v}_{1}, \mathrm{v}_{2}, \ldots, \mathrm{v}_{\mathrm{k}}$ is $\left(\begin{array}{l}k \\ 2\end{array}\right)$. Hence in $\mathrm{Kc}_{\mathrm{n}}(\mathrm{k})$ there are $\left(\begin{array}{l}k \\ 2\end{array}\right)$ vertices are at of distance 2 and the remaining $\left(\begin{array}{l}n \\ 2\end{array}\right)-\left(\begin{array}{l}k \\ 2\end{array}\right)$ vertices are at of distance 1.Therefore

$$
\sum_{\substack{i, j=1 \\
i<j}}^{n} d^{2}\left(v_{i}, v_{j}\right)=\left(\left(\begin{array}{l}
n \\
2
\end{array}\right)-\left(\begin{array}{l}
k \\
2
\end{array}\right)\right) \cdot 1+\left(\begin{array}{l}
k \\
2
\end{array}\right)(2)^{2} \text {. Hence the result. }
$$

II. For $\mathrm{Kc}_{\mathrm{n}}(\mathrm{k})$, number of removed edges are $\mathrm{k}(\mathrm{k}-1) / 2$ and sum of common entries is

$$
\frac{k(k-1)}{4}\left\{\frac{k(k-1)}{2}-1-2(k-2)\right\}+\left(\frac{k(k-1)}{2}\right)
$$

Therefore from (i) we get

$$
\begin{aligned}
& \mathrm{W}_{\mathrm{e} 0}\left(\mathrm{Kc}_{\mathrm{n}}(\mathrm{k})\right)=\mathrm{W}_{\mathrm{e} 0}\left(\mathrm{~K}_{\mathrm{n}}\right)-\frac{k(k-1)}{2}[\mathrm{n}(\mathrm{n}-1)-2 \mathrm{n}+2]+\frac{k(k-1)}{4}\left\{\frac{k(k-1)}{2}-1-2(k-2)\right\} \\
& -2(k-2)\}+\left(\begin{array}{l}
\frac{k(k-1)}{2} \\
2
\end{array}\right)
\end{aligned}
$$

Hence the result. 
III. For $\mathrm{Kc}_{\mathrm{n}}(\mathrm{k})$ sum of square of common entries in $\mathrm{D}_{1}$ is

$$
\frac{3 k(k-1)}{4}\left\{\frac{k(k-1)}{2}-1-2(k-2)\right\}+\left(\frac{k(k-1)}{2}\right)
$$

From (ii) we get

$$
\begin{aligned}
& W_{e 0}^{d^{2}}\left(K c_{n}(\mathrm{k})\right)=W_{e 0}^{d^{2}}\left(K_{n}\right)-\frac{k(k-1)}{2}\{2 \mathrm{n}(\mathrm{n}-1)-6(\mathrm{n}-2)-4\}+ \\
& +\frac{3 k(k-1)}{4}\left\{\frac{k(k-1)}{2}-1-2(k-2)\right\}+\left(\frac{k(k-1)}{2}\right)
\end{aligned}
$$

Hence the result.

For $\mathrm{Kc}_{\mathrm{n}}(\mathrm{k})$ sum and sum of square of common entries in $\mathrm{D}_{4}$ is $\left(\frac{k(k-1)}{2}\right)$

Hence IV and V follows from (i) and (ii)

Theorem 2.7

$\mathrm{W}\left(\mathrm{Ka}_{\mathrm{n}}(\mathrm{m}, \mathrm{k})\right)=\left(\begin{array}{c}n \\ 2\end{array}\right)+k\left(\begin{array}{c}m \\ 2\end{array}\right) \quad[\mathbf{8}]$

Theorem 2.8

I. $\quad \mathrm{WW}\left(\mathrm{Ka}_{\mathrm{n}}(\mathrm{m}, \mathrm{k})\right)=\mathrm{n}(\mathrm{n}-1)+2 \mathrm{~km}(\mathrm{~m}-1)$

II. $\quad \mathrm{W}_{\mathrm{e} 0}\left(\mathrm{Ka}_{\mathrm{n}}(\mathrm{m}, \mathrm{k})\right)=\frac{n(n-1)^{2}(n-2)}{4}-k \frac{m(m-1)}{8}\{4(n-2)(n-1)-(m-2)(m-3)-k m(m-1)+$ $+2+m(m-1) 2 k$

III. $\quad \mathrm{WW}_{\mathrm{e} 0}\left(\mathrm{Ka}_{\mathrm{n}}(\mathrm{m}, \mathrm{k})\right)=\frac{n(n-1)(n-2)(3 n-5)}{4}-k \frac{m(m-1)}{4}\left\{2\left(3 n^{2}-11 n+10\right)-2(m-2)(m-3)-\right.$ $-k m m-1+2+4 m(m-1) 2 k$

IV. $\quad \mathrm{W}_{\mathrm{e} 4}\left(\mathrm{Ka}_{\mathrm{n}}(\mathrm{m}, \mathrm{k})\right)=\frac{n(n-1)(n-2)(n+1)}{8}-\frac{k m(m-1)}{8}\{2 n(n-1)-k m(m-1)-2\}$

V. $\quad \mathrm{WW}_{\mathrm{e} 4}\left(\mathrm{Ka}_{\mathrm{n}}(\mathrm{m}, \mathrm{k})\right)=\frac{n(n-1)(n-2)(n+1)}{4}-\frac{k m(m-1)}{4}\{2 n(n-1)-k m(m-1)-2\}$

Proof:

There are $\mathrm{k}$ independent complete subgraphs $\mathrm{K}_{\mathrm{m}}$. Therefore, the number ofedges removed from $\mathrm{K}_{\mathrm{n}}$ will be

$$
k\left(\begin{array}{c}
m \\
2
\end{array}\right) \text { Hence } \sum_{\substack{i<j \\
i, j=1}}^{n} d^{2}\left(v_{i}, v_{j}\right)=W\left(K a_{n}(m, k)\right)+\left(\left(\begin{array}{c}
n \\
2
\end{array}\right)-k\left(\begin{array}{c}
m \\
2
\end{array}\right)\right) \cdot 1+k\left(\begin{array}{c}
m \\
2
\end{array}\right) \cdot 2^{2}
$$

Therefore $\mathrm{WW}\left(\mathrm{Ka}_{\mathrm{n}}(\mathrm{m}, \mathrm{k})\right)=\mathrm{n}(\mathrm{n}-1)+2 \mathrm{~km}(\mathrm{~m}-1)$

For $\mathrm{Ka}_{\mathrm{n}}(\mathrm{m}, \mathrm{k})$, sum of common entries in $\mathrm{D}_{1}$ is

$$
\left(\frac{k m(m-1)}{2}\right)+\frac{1}{2}\left\{\frac{k m(m-1)}{2}\left[\frac{m(m-1)}{2}-1-2(m-2)\right]\right\}+\left(\frac{m(m-1)}{2}\right)^{2}
$$

and sum of square of common entries is

$$
\left(\frac{k m(m-1)}{2}\right)+\frac{1}{2}\left\{\frac{3 k m(m-1)}{2}\left[\frac{m(m-1)}{2}-1-2(m-2)\right]\right\}+3\left(\frac{m(m-1)}{2}\right)^{2}
$$

Hence the results II and III follows from (i) and (ii)

For $\mathrm{Ka}_{\mathrm{n}}(\mathrm{m}, \mathrm{k})$, sum and sum of square of common entries in $\mathrm{D}_{4}$ is $\left(\begin{array}{c}\frac{k m(m-1)}{2} \\ 2\end{array}\right)$.

Therefore from (i) and (ii) we get

$$
\begin{aligned}
& \mathrm{W}_{\mathrm{e} 4}\left(\mathrm{Ka}_{\mathrm{n}}(\mathrm{m}, \mathrm{k})\right)=\frac{n(n-1)(n-2)(n+1)}{8}-\frac{k m(m-1)}{8}\{2 n(n-1)-k m(m-1)-2\} \\
& \mathrm{WW}_{\mathrm{e} 4}\left(\mathrm{Ka}_{\mathrm{n}}(\mathrm{m}, \mathrm{k})\right)=\frac{n(n-1)(n-2)(n+1)}{4}-\frac{k m(m-1)}{4}\{2 n(n-1)-k m(m-1)-2\}
\end{aligned}
$$




\section{Cluster Graphsobtain From Bipartite Graphs} graph $\mathrm{K}_{\mathrm{m}, \mathrm{n}}$.

Here also we consider different types of graphs obtained by deleting edges from the complete bipartite

DEFINITION 3.1.Let $e_{i}, i=1,2, \ldots, k, 1 \leq k \leq \min \{m, n\}$ be independent edgesof the complete graph $\mathrm{K}_{\mathrm{m}, \mathrm{n}} \mathrm{m}, \mathrm{n} \geq 1$. The graph $\mathrm{Ka}_{\mathrm{m}, \mathrm{n}}(\mathrm{k})$ is obtained by deleting $\mathrm{e}_{\mathrm{i}}, \mathrm{i}=1$ to $\mathrm{k}$ edges from $\mathrm{K}_{\mathrm{m}, \mathrm{n}}$. Also, $\mathrm{Ka}_{\mathrm{m}, \mathrm{n}}(0) \equiv \mathrm{K}_{\mathrm{m}, \mathrm{n}}$.

DEFINITION 2.3.If $\mathrm{K}_{\mathrm{r}, \mathrm{s}}$ is any subgraph of $\mathrm{K}_{\mathrm{m}, \mathrm{n}}, 1 \leq \mathrm{r}<\mathrm{m}, 1 \leq \mathrm{s}<\mathrm{n}$ andKb $\mathrm{K}_{\mathrm{m}, \mathrm{n}}(\mathrm{r}, \mathrm{s})$ is the graph obtained by deleting the edges of $\mathrm{K}_{\mathrm{r}, \mathrm{s}}$ from $\mathrm{K}_{\mathrm{m}, \mathrm{n}}$. In addition, $\mathrm{Kb}_{\mathrm{m}, \mathrm{n}}(0,0) \equiv \mathrm{Kb}_{\mathrm{m}, \mathrm{n}}(\mathrm{r}, 0) \equiv \mathrm{Kb}_{\mathrm{n}}(0, \mathrm{~s}) \equiv \mathrm{K}_{\mathrm{m}, \mathrm{n}}$.

Theorem 3.1

$\mathrm{W}\left(\mathrm{Ka}_{\mathrm{m}, \mathrm{n}}(\mathrm{k})\right)=\mathrm{W}\left(\mathrm{K}_{\mathrm{m}, \mathrm{n}}\right)+2 \mathrm{k}$

Theorem 3.2

I. $\quad W W\left(K_{m, n}(k)\right)=3(m+n)^{2}-3(m+n)-4 m n+10 k$

II. $\quad \mathrm{W}_{\mathrm{e} 0}\left(\mathrm{Ka}_{\mathrm{m}, \mathrm{n}}(\mathrm{k})\right)=\left(\frac{n m}{2}-k\right)(2 n m-n-m)+\left(\begin{array}{c}k \\ 2\end{array}\right)$

III. $\quad \mathrm{WW}_{\mathrm{e} 0}\left(\mathrm{Ka}_{\mathrm{m}, \mathrm{n}}(\mathrm{k})\right)=(\mathrm{nm}-2 \mathrm{k})(3 \mathrm{~nm}-2(\mathrm{n}+\mathrm{m})+1)+\mathrm{k}(\mathrm{k}-1)$

IV. $\quad \mathrm{W}_{\mathrm{e} 4}\left(\mathrm{Ka}_{\mathrm{m}, \mathrm{n}}(\mathrm{k})\right)=(\mathrm{nm}-1)(\mathrm{nm}-2 \mathrm{k})+\mathrm{k}(\mathrm{k}-1)$

V. $\quad \mathrm{WW}_{\mathrm{e} 4}\left(\mathrm{Ka}_{\mathrm{m}, \mathrm{n}}(\mathrm{k})\right)=3\{(\mathrm{~nm}-1)(\mathrm{nm}-2 \mathrm{k})+\mathrm{k}(\mathrm{k}-1)\}$

Proof:

I. Number of edges in $\mathrm{K}_{\mathrm{m}, \mathrm{n}}$ is $\mathrm{mn}$. Removal of an edge from $\mathrm{Km}, \mathrm{n}$ will increase its Wiener number by 2.Therefore half sum of square of distance between all pair of vertices of $\mathrm{K}_{\mathrm{m}, \mathrm{n}}$ will increase by 8 .Hence for $\mathrm{Ka}_{\mathrm{m}, \mathrm{n}}(\mathrm{k}), \mathrm{WW}\left(\mathrm{Ka}_{\mathrm{m}, \mathrm{n}}(\mathrm{k})\right)=\mathrm{WW}\left(\mathrm{Ka}_{\mathrm{m}, \mathrm{n}}\right)+10 \mathrm{k}=3(\mathrm{~m}+\mathrm{n})^{2}-3(\mathrm{~m}+\mathrm{n})-4 \mathrm{mn}+10 \mathrm{k}$.

II. For $\mathrm{Ka}_{\mathrm{m}, \mathrm{n}}(\mathrm{k})$,number of edges removed from $\mathrm{K}_{\mathrm{n}, \mathrm{m}}$ is $\mathrm{k}$ and row(column) sum of $\mathrm{D}_{1}$ is $2 \mathrm{mn}-\mathrm{n}-\mathrm{m}$ since number of 2's in each row(column) is mn-1-(n+m-2) and number of 1's is $m+n-2$ and the sum of common entries is $\left(\begin{array}{c}k \\ 2\end{array}\right)$. Therefore $\mathrm{W}_{\mathrm{e} 0}\left(\mathrm{Ka}_{\mathrm{m}, \mathrm{n}}(\mathrm{k})\right)=\mathrm{W}_{\mathrm{e} 0}\left(\mathrm{~K}_{\mathrm{m}, \mathrm{n}}(\mathrm{k})\right)-\mathrm{k}(2 \mathrm{mn}-\mathrm{m}-\mathrm{m})+\left(\begin{array}{c}k \\ 2\end{array}\right)$. Hence the result.

III. For $\mathrm{Ka}_{\mathrm{m}, \mathrm{n}}(\mathrm{k})$, sum of square of entries in a row (column) of $\mathrm{D}_{1}$ is $4 \mathrm{mn}-3 \mathrm{n}-3 \mathrm{~m}+2$ and sum of square of common entries is $\left(\begin{array}{l}k \\ 2\end{array}\right)$.Therefore

$$
W_{e 0}^{d^{2}}\left(K a_{m, n}(\mathrm{k})\right)=W_{e 0}^{d^{2}}\left(K a_{n}, m\right)-k\{4 \mathrm{mn}-3 \mathrm{n}-3 \mathrm{~m}+2\}+\left(\begin{array}{c}
k \\
2
\end{array}\right)
$$

Hence $\mathrm{WW}_{\mathrm{e} 0}\left(\mathrm{Ka}_{\mathrm{m}, \mathrm{n}}(\mathrm{k})\right)=(\mathrm{nm}-2 \mathrm{k})(3 \mathrm{~nm}-2(\mathrm{n}+\mathrm{m})+1)+\mathrm{k}(\mathrm{k}-1)$

IV. Second edge distance matrix, $\mathrm{D}_{4}=\left(\right.$ dij)of $\mathrm{K}_{\mathrm{n}, \mathrm{m}}$ is a $\mathrm{nm} X \mathrm{~nm}$ matrix in which all entries are two except the diagonal .Hence row(column) sum of $\mathrm{D}_{4}$ is $2(n m-1)$ and for $\mathrm{Ka}_{\mathrm{n}, \mathrm{m}}(\mathrm{k})$ sum of common entries is $2\left(\begin{array}{l}k \\ 2\end{array}\right)$. Therefore $\mathrm{W}_{\mathrm{e} 4}\left(\mathrm{Ka}_{\mathrm{m}, \mathrm{n}}(\mathrm{k})\right)=\mathrm{W}_{\mathrm{e} 4}\left(\mathrm{~K}_{\mathrm{m}, \mathrm{n}}(\mathrm{k})\right)-\mathrm{k}\left(2(\mathrm{mn}-1)+2\left(\begin{array}{l}k \\ 2\end{array}\right)\right.$. Hence the result.

V. Sum of square of entries in a row (column) in $\mathrm{D}_{4}$ is $4(n m-1)$ and sum of square of common entries is $4\left(\begin{array}{l}k \\ 2\end{array}\right)$. Hence the result.

Theorem:3.3

$\mathrm{W}\left(\mathrm{Kb}_{\mathrm{m}, \mathrm{n}}(\mathrm{r}, \mathrm{s})\right)=\mathrm{W}\left(\mathrm{K}_{\mathrm{m}, \mathrm{n}}\right)+2 \mathrm{rs}$.

Theorem 3.4

I. $\quad \mathrm{WW}\left(\mathrm{Kb}_{\mathrm{m}, \mathrm{n}}(\mathrm{r}, \mathrm{s})\right)=3(\mathrm{~m}+\mathrm{n})^{2}-3(\mathrm{~m}+\mathrm{n})-4 \mathrm{mn}+10 \mathrm{rs}$

II. $\quad \mathrm{W}_{\mathrm{e} 0}\left(\mathrm{~Kb}_{\mathrm{m}, \mathrm{n}}(\mathrm{r}, \mathrm{s})\right)=\left(\frac{n m}{2}-r s\right)(2 n m-n-m)+\frac{r s}{2}(2 r s-r-s)$

III. $\quad \mathrm{WW}_{\mathrm{e} 0}\left(\mathrm{~Kb}_{\mathrm{m}, \mathrm{n}}(\mathrm{r}, \mathrm{s})\right)=(\mathrm{nm}-2 \mathrm{rs})(3 \mathrm{~nm}-2(\mathrm{n}+\mathrm{m})+1)+\mathrm{rs}(3 \mathrm{rs}-2 \mathrm{r}-2 \mathrm{~s}+1)$

IV. $\quad \mathrm{W}_{\mathrm{e} 4}\left(\mathrm{~Kb}_{\mathrm{m}, \mathrm{n}}(\mathrm{r}, \mathrm{s})\right)=(\mathrm{nm}-1)(\mathrm{nm}-2 \mathrm{rs})+\mathrm{rs}(\mathrm{rs}-1)$

V. $\quad \mathrm{WW}_{\mathrm{e} 4}\left(\mathrm{~Kb}_{\mathrm{m}, \mathrm{n}}(\mathrm{r}, \mathrm{s})\right)=3\{(\mathrm{~nm}-1)(\mathrm{nm}-2 \mathrm{rs})+\mathrm{rs}(\mathrm{rs}-1)\}$

Proof:

For $\mathrm{Kb}_{\mathrm{m}, \mathrm{n}}(\mathrm{r}, \mathrm{s})$, number of edges removed from $\mathrm{K}_{\mathrm{n}, \mathrm{m}}$ is rs and sum of common entries in $\mathrm{D}_{1}$ is $\left(\begin{array}{c}r s \\ 2\end{array}\right)+\frac{1}{2}(r s-$ $r-s+1)$ and sum of square of common entries is $\left(\begin{array}{c}r s \\ 2\end{array}\right)+\frac{3}{2}(r s-r-s+1)$. Then the proof is as similar as before.

\section{Conclusion} graphs.

Hence we have calculated the a vertex and edge hyper Wiener number of some complete and bipartite 


\section{References}

[1] Trinajsti N., Chemical Graph Theory, CRC Press, Boca Raton, FL, 1983; 2nd revised edition, 1992.

[2] Wiener. H., Structural determination of parafinboiling, J. Am. Chem . Soc. 69 , (1947) 17-20.

[3] Randic,M., Novel molecular descriptor for structure property studies .Chem. Phys. Lett. 1993,211,478-483

[4] Klein, D.J.; Lukovits, I.; Gutman, I., On the definition of the hyper -Wiener index for cycle - containing structures. J.Chem. Inf. Comput. Sci. 1995,35,50-52.

[5] Ali Iranmanesh ,Gutman . I , OmidKhormali, Mahmian .A , The edge version of Wiener Index , MATCH Commun.Math.Comput.Chem ., 61 (2009), $663-672$

[6] Ali Iranmanexh, SoltaniKafrani.A ., OmidKhormali, A New Version of Hyper - Wiener Index , MATCH Commun.Math.Comput.Chem ., 65 (2011), $113-122$

[7] Gutman.I,Pavlovic,, The energy of some graphs with large number of edges,Bull.Acade.Serbe.Sci.Arts (cl.Math, Natur),118,1999,35-39

[8] Sridharan.N,Thilakam.K, Some cluster graphs, AntarticaJ.Math. ,9(5),2012,445-450

\section{Examples follow:}

Journal Papers:

[1] M Ozaki, Y. Adachi, Y. Iwahori, and N. Ishii, Application of fuzzy theory to writer recognition of Chinese characters, International Journal of Modelling and Simulation, 18(2), 1998, 112-116. (8)

Note that the journal title, volume number and issue number are set in italics.

Books:

[2] R.E. Moore, Interval analysis (Englewood Cliffs, NJ: Prentice-Hall, 1966). (8)

Note that the title of the book is in lower case letters and italicized. There is no comma following the title. Place of publication and publisher are given.

\section{Chapters in Books:}

[3] P.O. Bishop, Neurophysiology of binocular vision, in J.Houseman (Ed.), Handbook of physiology, 4 (New York: Springer-Verlag, 1970) 342-366. (8)

Note that the place of publication, publisher, and year of publication are enclosed in brackets. Editor of book is listed before book title.

\section{Theses:}

[4] D.S. Chan, Theory and implementation of multidimensional discrete systems for signal processing, doctoral diss., Massachusetts Institute of Technology, Cambridge, MA, 1978. (8)

Note that thesis title is set in italics and the university that granted the degree is listed along with location information

\section{Proceedings Papers:}

[5] W.J. Book, Modelling design and control of flexible manipulator arms: A tutorial review, Proc. 29th IEEE Conf. on Decision and Control, San Francisco, CA, 1990, 500-506(8) 